\title{
Guía para la implementación de un sistema de gestión integral en la empresa Óptima de Urabá S. A. E.S.P*
}

\section{Guide for the implementation of a comprehensive management system in the company Óptima de Urabá S. A. E.S.P.}

\section{Guia para a implementação de um sistema de gestão inteǵral na empresa Óptima de Úraba S. A. E.S.P}

Recibido: 25 de septiembre de 2017

Revisado: 15 de enero de 2018

Aceptado: 23 de marzo de 2018

Ana María Estrada Tordecilla*

Consultora independiente

\section{RESUMEN}

Este artículo presenta los resultados de una investigación cuyo objetivo fue elaborar una guía para la implementación de un sistema de gestión integral en la empresa Óptima de Urabá S. A. E.S.P., con base a las normas ISO 9001:2015, ISO 14001:2015 y
OSHAS18001:2007, como respuesta al fortalecimiento de las políticas estratégicas en materia de gestión que garanticen un desarrollo eficaz y sostenible dentro de la organización, a través de la aplicación de dos herramientas cuantitativas que proporcionan el diagnóstico actual de la organización y los elementos bases para la construcción de la guía en mención. Los resultados de

Artículo de resultado de investigación. DOI: http://dx.doi.org/10.15332/s2145-1389.2018.0001.04

** Magíster en Calidad y Gestión Integral. Ingeniera Sanitaria y Ambiental. Consultora independiente. Correo electrónico: anamariaestrada@hotmail.es 
la investigación reflejan la necesidad de introducir el manejo de temas ambientales y de seguridad laboral dentro de la organización, para abordar un esquema integral en el sistema de gestión actual. Se presenta una visión general de los elementos bases de integración, que deben estar alineados con la propuesta de la guía del sistema de gestión integral y ajustados al sistema de gestión de calidad existente en la organización objeto de estudio, aportando de esta forma, nuevas iniciativas de integración en organizaciones agremiadas al sector de servicios públicos.

Palabras clave: gestión, revisión de cuentas, innovación e invención, procesos e incentivos, modelos de planificación, política de planificación, planificación, coordinación y reforma, sistema integrado de gestión.

\section{ABSTRACT}

This article presents the results of a research whose objective was to develop a guide for the implementation of a comprehensive management system in the company Óptima de Urabá S. A. E.S.P., based on the standards ISO 9001:2015, ISO 14001:2015 and OSHAS18001:2007, as response to the strengthening of strategic policies in management issues that ensure an effective and sustainable development within the organization, through the application of two quantitative tools that provide the current diagnosis of the organization and the basic elements for the construction of the mentioned guide. The results of the research reflect the need to introduce the management of environmental and occupational safety issues in the organization, to address a comprehensive scheme in the current management system. An overview of the basic elements of integration is presented, which must be aligned with the proposal of the comprehensive management system guide and adjusted to the existing quality management system in the organization under study, thus contributing with new initiatives of integration in organizations associated to the public services sector.

Keywords: Management, review of accounts, innovation and invention, processes and incentives, planning models, planning policy, planning, coordination and reform, integrated management system.

\section{RESUMO}

Este artigo apresenta os resultados de uma investigação cujo objetivo foi elaborar um guia para a implementação de um sistema de gestão integral na empresa Óptima de Urabá, com base nas normas ISO 9001: 2015, ISO 14001: 2015 e OSHAS18001: 2007, como resposta ao fortalecimento das políticas estratégicas em matéria de gestão que garantam um desenvolvimento eficaz e sustentável dentro da organização, através da aplicação de duas ferramentas quantitativas que proporcionam o diagnóstico atual da organização e os elementos de base para a construção do guia em menção. Os resultados da investigação refletem a necessidade de introduzir o manejo de temas ambientais e segurança laboral dentro da organização, para abordar um esquema integral no sistema de gestão atual. se apresenta uma visão geral dos elementos fundamentais de integração, que devem ser alinhadas com a proposta do guia do sistema de gestão integral e ajustados ao sistema de gestão da qualidade existente na organização objeto de estudo, contribuindo desta forma, novas iniciativas de integração em organizações associadas ao setor de serviços públicos.

Palavras-chave: gestão, revisão de contas, inovação e invenção, processos e incentivos, modelos de planificação, política de planificaçãoo: planificação, coordenação e reforma, sistema integrado de gestão. 


\section{INTRODUCCIÓN}

Los sistemas de gestión se han popularizado a nivel mundial por los múltiples beneficios a las condiciones laborales, productivas, económicas y ambientales de las organizaciones; esta fuerza sigue incrementándose puesto que "el número de organizaciones certificadas con ISO 9001 aumentó en todo el mundo, pasando de 27.000 en 1993 a más de 520.000 a enero de 2004, seguido por las certificaciones en las normas ISO 14001 y OHSAS 18001" (Benavides, 2013, p. 75). Su implementación se encuentra sujeta a cambios sociales, económicos y políticos, que hoy en día sufren las sociedades; esto hace que las organizaciones enfrenten nuevos retos que conlleven a satisfacer sus propias necesidades y la de sus partes interesadas.

La integración de estos sistemas también vienen cobrando importancia a nivel mundial; tomando como referente teórico un estudio realizado por Salomone en organizaciones italianas, se encuentra que la implementación de sistemas integrados de gestión en las empresas en diferentes sectores socioeconómicos generan numerosos beneficios, los más destacados son la optimización, unificación de la auditoría, tanto interna (78\%) y externa (65\%), la reducción en la mayor parte de la documentación (69\%), y en general, todas las áreas en las que las sinergias entre los sistemas podrín ser explotados, lo que ahorra tiempo, dinero y mano de obra (Salomone, 2011).

Sin embargo, muchas de las empresas que implementan sistemas integrados de gestión encuentran dificultades en la elección de un modelo que logre cohesionar sus interacciones, y como consecuencia terminan manejando un sistema de gestión fraccionado sin ningún tipo de relación, lo que conlleva al fracaso del sistema de gestión. Según Morín (1994), la mayor parte de los sistemas no están conformados por partes, elementos 0 constituyentes, sino por acciones entre unidades complejas cuyo conjunto constituye la organización del sistema, estas acciones deben estar mediatizadas por interacciones, para dar mayor respaldo a los procesos ejecutados.

De acuerdo con lo anterior, se hace necesario trascender los sistemas de gestión a un enfoque integral, donde se logre la comprensión de la organización desde las interacciones y dinámicas que se desarrollan con la articulación de la estructura del negocio, el direccionamiento estratégico, las culturas de la organización y la integración de los diferentes sistemas de gestión que la organización dispone para implementar su estrategia (Losada y Guarín, 2009). Las organizaciones de servicios públicos domiciliarios no son ajenas a la articulación de los sistemas de gestión, por el contrario, deben garantizar una buena calidad en la prestación del servicio.

En la investigación realizada por Valencia, Madrid y Tabares (2014), afirman que la experiencia de la mayoría de las empresas de servicios públicos indica que los elevados valores del agua no contabilizada se originan en las deficiencias de la gestión comercial, principalmente por problemas de facturación, recaudación de pagos y políticas inadecuadas en materia de cuentas vencidas, y no se deben solamente al alto porcentaje de pérdidas por fugas en los sistemas de distribución. Esto refleja una mala gestión estratégica en los sistemas implementados, que a pesar de la existencia de estas herramientas, la gestión de calidad, ambiente y en seguridad y salud en el trabajo dentro las organizaciones, no se encuentran consolidados en su totalidad; es por esto que las organizaciones de servicios públicos deben acoger la gestión integral como un enfoque estratégico que direccione el mejoramiento continuo de los sistemas de gestión de la calidad, ambiente y seguridad y salud en el trabajo.

En este contexto, Atehortúa, Bustamante y Valencia (2008), proponen un modelo de SGI que recoge elementos del modelo American Productivity and Quality 
Center (APQC) de los Estados Unidos y el modelo de la NTCGP 1000:2004, pero modifica algunas denominaciones para hacerlas más compatibles con las normas de gestión, en este modelo, cada proceso es un subsistema del sistema de gestión integral. Igualmente, expresan que los elementos o enfoques comunes a los modelos de gestión normalizados son: enfoque por procesos, enfoque de las partes interesadas, enfoque en cumplimiento de la normatividad, enfoque en riesgos, enfoque en la documentación, enfoque en las competencias, enfoque en seguimiento, medición y mejora, los cuales sirven como fundamento conceptual para la propuesta de la guía.

De esta forma, el proyecto de investigación cobra importancia en el aporte de conocimiento sobre el manejo que actualmente le está dando la empresa Óptima de Urabá a su sistema de gestión de la calidad, y refleja la necesidad de diseñar una guía para la implementación de un sistema de gestión integral, donde se logre obtener mayor control en la reducción de impactos, mejoras en el rendimiento de los procesos y en la calidad de vida de sus partes interesadas; además de generar iniciativas en las demás organizaciones agremiadas al sector de servicios públicos domiciliarios, en acoger e implementar esta propuesta de manera satisfactoria dentro de su estructura interna.

La presente investigación contempla un enfoque cuantitativo, que parte de una base teórica analizando los problemas detectados mediante la revisión de la literatura existente y la implementación de dos herramientas de diagnóstico, para llegar a la obtención de los resultados que revelan las necesidades reales de la organización. De esta forma, se obtienen las bases para la construcción de la guía de sistema de gestión integral para la empresa Óptima de Urabá, la cual incluye un proceso de validación por medio de expertos en sistemas de gestión integral, quienes otorgan criterios de eficacia y efectividad para su proceso de implementación, permitiéndole a la organización articular los requisitos de las normas ISO 9001, ISO 14001 y OSHAS 18001 en una sola gestión.

\section{METODOLOGÍA}

La investigación parte de una base teórica que analiza los problemas detectados mediante la revisión de la literatura existente y la implementación de dos herramientas de diagnóstico (uno tipo Likert y otro dicotómico), elaborados a partir de los elementos del marco teórico, los cuales son abordados desde un enfoque cuantitativo con un apoyo cualitativo incluido en una de las herramientas de diagnóstico.

Una vez ejecutadas las herramientas de diagnóstico, se llega a la obtención de los resultados que revelan datos concretos y hechos reales de la organización, los cuales permiten diseñar la guía para la implementación de un sistema integral de gestión alineada a las necesidades de la empresa Óptima de Urabá. Una vez diseñada la guía se hace la validación por medio de expertos en sistemas de gestión integral.

Con el propósito de profundizar sobre los aspectos relacionados con la metodología, se implementa un diseño metodológico no experimental, el cual propone realizar la investigación sin manipular deliberadamente las variables, es decir, hacer estudios en los que no se hace variar en forma intencional las variables independientes para ver su efecto en sobre las otras variables (Sampieri, Fernándezy Baptista, 2014, p. 152). Igualmente se hace de forma transversal, recolectando datos en un solo momento, para describir variables y analizar su incidencia e interrelación en un momento dado.

Para el desarrollo de la hipótesis planteada: "Eficacia de la guía para la implementación de un SGI para la empresa Óptima de Urabá", la investigación acoge 
el paradigma empírico-analítico en miras de orientar la investigación hacia la comprobación de la hipótesis explicando y prediciendo las conductas de los sujetos implicados en la organización objeto de estudio. Como estrategia para la verificación de la hipótesis, se contempla implementar un proceso de validación de la guía por medio de expertos en SGI, en este proceso se establece como criterio de validación la eficacia de la guía.

Así mismo, la investigación aborda las variables descritas en la tabla 1, las cuales son definidas teniendo en cuenta la base conceptual establecida, con el propósito de medir el nivel de gestión en el que se encuentra actualmente la organización, y con ello poder comprobar la hipótesis con la construcción y validación de la guía, por lo cual estas variables son el fundamento sobre el que se construyen los enunciados de las herramientas de diagnóstico.

Para la recopilación de la información, se aplican dos herramientas de diagnóstico: una encuesta tipo Likert construida a partir del análisis de contenido y otro tipo dicotómico con preguntas cerradas. La información se evalúa usando la técnica de estadística descriptiva.

La población objeto de estudio corresponde al personal de planta de la empresa Óptima de Urabá. La muestra fue calculada bajo el software Decisión Analyst STATS 2.0, con un error máximo aceptable

Tabla 1. Definición de variables

\begin{tabular}{|c|c|c|}
\hline Indicador & Variables & Definición \\
\hline \multirow{10}{*}{$\begin{array}{l}\text { Gestión integral } \\
(9001,14001,18001)\end{array}$} & $\begin{array}{l}\text { Contexto integral de la } \\
\text { organización }\end{array}$ & $\begin{array}{l}\text { Gestión de una organización que obedece a una sola unidad de propósito (visión de la } \\
\text { organización) y a una sola naturaleza (la misión). }\end{array}$ \\
\hline & Liderazgo & $\begin{array}{l}\text { Los líderes que establecen la unidad de propósito y la dirección de la organización, y } \\
\text { crean condiciones en las que las personas se involucran en el logro de los objetivos de la } \\
\text { organización. }\end{array}$ \\
\hline & Procesos & $\begin{array}{l}\text { Implica para el sistema de gestión integral asuntos como la identificación, descripción, } \\
\text { documentación, implementación, evaluación y mejora de los diferentes procesos. }\end{array}$ \\
\hline & Partes interesadas & $\begin{array}{l}\text { Son aquellas que generan riesgo significativo para la sostenibilidad de la organización si } \\
\text { sus necesidades y expectativas no se cumplen. }\end{array}$ \\
\hline & Normatividad & Cumplimiento de los requisitos legales, en las normas de gestión. \\
\hline & Riesgos & $\begin{array}{l}\text { Requisito implícito o explícito que hace que el SGI adquiera un carácter preventivo } \\
\text { y proactivo, teniendo en cuenta las partes interesadas en la ocurrencia de los posibles } \\
\text { riesgos. }\end{array}$ \\
\hline & Comunicación & $\begin{array}{l}\text { La comunicación interna planificada y eficaz, y la externa, fomentan el compromiso de } \\
\text { las personas y aumenta la comprensión del contexto de la organización, las necesidades y } \\
\text { expectativas de los consumidores y otras partes interesadas, y el sistema de gestión. }\end{array}$ \\
\hline & Documentación & $\begin{array}{l}\text { Contribuye al propósito de la gestión del conocimiento y a tener base material sobre la } \\
\text { cual introducir mejoras e innovaciones a los procesos. }\end{array}$ \\
\hline & Competencias & $\begin{array}{l}\text { Aplicación de habilidades, formación, educación y experiencia necesarias para } \\
\text { desempeñar sus roles y responsabilidades. }\end{array}$ \\
\hline & Seguimiento y mejora & $\begin{array}{l}\text { Determinación del estado de un sistema, un proceso, un producto, un servicio o una } \\
\text { actividad, que dan las bases a la implementación de actividades de mejora al SGI. }\end{array}$ \\
\hline
\end{tabular}

Fuente: elaboración propia. 
del $5 \%$, porcentaje estimado de la muestra del $50 \%$ y un nivel deseado de confianza del $95 \%$. De acuerdo con los funcionarios involucrados en el sistema de gestión de la calidad, el software arroja que, de una población total de 17 trabajadores, se aplicará la primera herramienta de diagnóstico (cuestionario tipo Likert) a 16 trabajadores, los cuales incluyen a 5 líderes de los siguientes procesos: financiero, operativo, comercial, administrativo y gestión humana, y 11 trabajadores involucrados de manera directa con el sistema de gestión en calidad. Para la segunda herramienta de diagnóstico, se tomará como muestra a los integrantes de la alta dirección: gerente y director.

En cuanto al diseño de la guía para la implementación del sistema de gestión integral en la organización objeto de estudio, se realiza por medio de la triangulación de los hallazgos obtenidos en: 1) diagnóstico documental efectuado en el primer objetivo; 2) consulta a los líderes de gestión para identificar las necesidades de las organizaciones y 3) el análisis de la estructura de alto nivel y las normas NTC ISO 9001, NTC ISO 14001 y OSHAS 18001, de esta forma se construye la guía del sistema de gestión integral. Por su parte, el proceso de validación de la guía diseñada es realizado a través de expertos en manejo de sistemas de gestión integral.

\section{RESULTADOS Y DISCUSIÓN}

En primer lugar, se describen los resultados del diagnóstico del estado actual del cumplimiento de las normas ISO 9001, ISO 14001 y OHSAS 18001, en la empresa Óptima de Urabá. En segundo lugar, se muestra la estructura de la guía diseñada para la implementación del sistema de gestión integral, que responde a las necesidades en gestión de la empresa objeto de investigación. En tercer lugar, se describen los hallazgos del proceso de validación de la guía diseñada para la empresa.

\section{Criterios para el análisis de datos}

En el análisis de resultados de las herramientas de diagnóstico, se tienen en cuenta los siguientes criterios de análisis de los datos:

- Cuestionario tipo Likert: sí el 60 \% de empleados responden a la afirmación entre "totalmente de acuerdo" y "de acuerdo", se considera que la misma se encuentra en un rango aceptable y se viene aplicando al interior de la organización, siempre y cuando la afirmación sea positiva, en caso de que la afirmación sea negativa se considera no aplicada. Si el $40 \%$ de empleados responden las variables "totalmente en desacuerdo", "en desacuerdo" y "neutro", la afirmación no se considera que se está promoviendo dentro de la organización y requiere una intervención a mediano o largo plazo.

- Cuestionario dicotómico: para el caso de las respuestas a este cuestionario, si ambas respuestas son contestadas afirmativamente (100\% de aceptación), significa que la intención de respuesta es acertada. Por el contrario, si alguna de las respuestas es negativa (50\% de aceptación), se considera que la variable requiere de intervención.

- En la evaluación de los enfoques o variables definidas, se evalúa el promedio de las afirmaciones positivas, las cuales se consideran acertadas si en ambas herramientas se supera el $80 \%$, de lo contrario son considerados procesos débiles que requieren de manera urgente líneas de acción al interior de la organización.

Para la evaluación de los enfoques o variables definidas, se calcula el promedio de las afirmaciones, la cuales se consideran acertadas si en las dos herramientas se obtiene un promedio superior al $80 \%$ de afirmaciones o preguntas positivas, de lo contrario son considerados procesos débiles que requieren de manera urgente líneas de acción al interior de la organización. 
De acuerdo con estos criterios, se realiza el análisis de los datos recolectados y se obtienen los resultados que facilitan, posteriormente, la construcción del diagnóstico actual de la organización con base a su sistema de gestión de la calidad.

\section{Hallazgoos del diagnóstico actual frente al sistema de gestión}

Teniendo en cuenta el análisis cuantitativo de los datos obtenidos en las herramientas de diagnóstico, se resumen los resultados en la tabla n. 2 .

Como se puede observar en los resultados, las variables contexto integral, procesos y liderazgo son las que requieren mayor atención al momento de implementar el sistema de gestión integral, sin embargo, se detallan observaciones y se consideran algunas medidas de acción para los demás enfoques.

Para dar un amplio análisis de los resultados en el enfoque contexto integral, se logra interpretar que dentro de la organización existe conocimiento del ámbito en el que se mueve la organización, sin embargo, se pueden presentar limitaciones en la comprensión del contexto a nivel local, nacional e internacional y en la cultura, conocimiento y desempeño organizacional, debido a que se maneja un bajo nivel de conocimiento de estos temas dentro de la organización.

Adicionalmente, no se reconocen la aplicabilidad y el alcance del SGG, tema que requiere una intervención para fortalecer la conceptualización, estructura, alcance, objetivos y requisitos del sistema, ya que a diferencia de los SGA y SGSST, el SGC debe aplicarse a toda la organización y debe tener un alcance definido que ayude a empoderar más a los empleados en este sistema de gestión. De esta forma, se evidencia la necesidad de implementar una gestión integral dentro de la organización, donde se contemplen los temas ambientales y de seguridad.

Para el enfoque de procesos, los resultados muestran que se identifican los procesos de comunicación interna y externa y los conductos regulares que deben aplicar al momento de realizar procesos de comunicación. Adicionalmente, se cuenta con claridad en las actividades que se deben desempeñar en cada proceso, los cuales se encuentran ajustados a los requisitos exigidos por la norma.

A pesar de que muchos de los empleados afirman que las características de los procesos se encuentran acordes al SGC, la estructura de los procesos debería

Tabla 2. Nivel de intervención de enfoques

\begin{tabular}{|c|c|c|c|c|}
\hline \multirow{2}{*}{ Enfoques } & \multicolumn{2}{|c|}{ Resultado herramientas } & \multirow{2}{*}{ Promedio } & \multirow{2}{*}{ Intervención } \\
\hline & Likert & Dicotómico & & \\
\hline Contexto integral de la organización & $68 \%$ & $40 \%$ & $54 \%$ & $\mathrm{Si}$ \\
\hline Procesos & $75 \%$ & $100 \%$ & $88 \%$ & No \\
\hline Partes interesadas & $74 \%$ & $100 \%$ & $87 \%$ & No \\
\hline Normatividad & $88 \%$ & $100 \%$ & $94 \%$ & No \\
\hline Riesgos & $63 \%$ & $100 \%$ & $81 \%$ & No \\
\hline Documentación & $88 \%$ & $100 \%$ & $94 \%$ & No \\
\hline Competencias & $96 \%$ & $100 \%$ & $98 \%$ & No \\
\hline Seguimiento y mejora & $93 \%$ & $85 \%$ & $89 \%$ & No \\
\hline Liderazgo & $66 \%$ & - & $66 \%$ & $\mathrm{Si}$ \\
\hline
\end{tabular}

Fuente: elaboración propia basada en los resultados obtenidos de las herramientas de diagnóstico. 
responder eficazmente a los requisitos exigidos. Por su parte, la alta dirección sugiere realizar fortalecimiento en la ejecución de los procesos, tema que es necesario tratarlo dentro de la guía.

Los empleados identifican de manera acertada los procesos de comunicación interna y externa, y los conductos regulares que deben aplicarse al momento de realizar procesos de comunicación. Adicionalmente, se cumple con los requisitos de las actividades posteriores a la prestación del servicio, se verifica el cumplimiento de todos los requisitos de calidad antes de prestar el servicio a los clientes y se evidencia que los procesos son planificados antes de la prestación del servicio y consideran que la realizan bajo condiciones controladas. A pesar de esto, los empleados no tienen claridad de los controles de seguridad que se le aplican a los contratistas, ni conocimiento en los controles a procesos de compra antes de prestar el servicio, por lo cual se hace necesario realizar una intervención en estos temas.

La alta dirección expresa que los procesos operativos, comerciales, de suministro y gestión humana implementados responden a los requisitos de la organización y que estos mismos se encuentran documentados, pero es importante que la organización sepa que no solo se trata de documentarlos, sino también de cómo gestionar los procesos a través de su manejo, implementación, evaluación y mejora, aspecto en el cual es necesario reforzar. Así mismo, consideran que la organización responde eficazmente a la gestión por procesos definida, sin embargo, es importante implementar estrategias de mejora en la ejecución de los procesos y garantizar que el modelo de gestión por procesos de la empresa sea revisado periódicamente para ajustarse al contexto en el que actúa, ya que es necesario ir alineando los procesos de acuerdo a los nuevos cambios que exige el sector.

Los resultados de este enfoque evidencian la necesidad de reforzar los temas en el manejo de controles operativos en la mayoría de los procesos desarrollados al interior de la organización, sin embargo, a pesar de que la organización tiene definido los procesos para responder a su sistema de gestión de calidad, es necesario involucrar los temas ambientales y de seguridad y salud en el trabajo.

Dentro de los resultados obtenidos para el enfoque de partes interesadas, se logra evidenciar que no se cuenta con suficiente claridad sobre los requisitos y expectativas de las partes interesadas, para lo cual se hace necesario formar principalmente a los operarios en las necesidades y funciones de las partes interesadas para apropiar los temas de requisitos y expectativas de las entidades que tienen interés específico en alguno en los ámbitos de desempeño de la organización.

Por su parte, los empleados consideran que se están implementando acciones eficaces en la comunicación con los clientes y vienen identificando los requisitos que se deben cumplir al momento de ofrecer el servicio, es evidente que muchos de ellos se encuentran en contacto directo con los clientes, lo que los obliga a prestar el servicio bajo condiciones controladas para asegurar el cumplimiento del servicio. Así mismo, la alta dirección considera que las partes interesadas se ven identificadas en cada uno de los ámbitos de desempeño de la organización, donde se encuentran relacionadas dentro del contexto social, económico, político y cultural; adicionalmente, expresan que la organización tiene en cuenta las exigencias de cada una de las entidades interesadas y los entes involucrados en cada proceso desarrollado dentro de la organización, lo que les permite obtener un equilibrio entre las necesidades y expectativas de estos mismos.

Dentro de la organización, se vienen aplicando criterios para la evaluación, selección, desempeño y reevaluación a los proveedores externos posterior al servicio prestado, y se hace control al cuidado de equipos y demás propiedades de los clientes y proveedores al momento de estar bajo el manejo de la organización. 
Por otro lado, el conocimiento de la empresa en cuanto a sus partes interesadas, solo está relacionada a la calidad en la operación del servicio, es necesario involucrar las entidades relacionadas con el medio ambiente y la seguridad laboral (autoridad ambiental, ARL, estado, clientes, proveedores, etc.) involucradas dentro de la organización, para lograr la integralidad de los procesos.

Para el enfoque de normatividad, los resultados reflejan que en la organización se tienen en cuenta los requisitos legales involucrados a los procesos, incluidos los ambientales y de seguridad laboral, esto es algo que juega a favor porque refleja manejo por parte de los empleados en temas ambientales y de seguridad. Adicionalmente, la organización identifica y evalúa el cumplimiento de los requisitos de los clientes, los legales, los implícitos y los de la organización antes de comprometerse a prestar el servicio, lo que refleja un buen equilibrio de intereses entre los clientes, proveedores, entidades de control y la organización.

Por su parte, la organización está comprometida y se interesa por cumplir los requisitos legales y normativos exigidos por las partes interesadas, reflejando que la organización opera bajo la normatividad que exige el sector de los servicios públicos domiciliarios. Así mismo, la organización identifica y cuenta con acceso oportuno a los requisitos legales que aplican a sus distintos ámbitos de actuación.

En términos generales, se puede destacar que la organización tiene compromiso en el cumplimiento de las obligaciones legales, aspecto importante que garantiza la prestación del servicio de acueducto, lo que se convierte en un buen factor para la efectividad de los sistemas de gestión.

En el enfoque en riesgos, se logra identificar que en la organización se determinan los riesgos y oportunidades en los procesos, con el fin de asegurar el logro de los resultados previstos en el SGC; sin embargo, en las respuestas ante emergencias ambientales y de SST, la mayoría de los empleados no cuentan con buena experiencia ni con conocimientos básicos para enfrentar eventualidades que requieran atención de emergencia (ambiental o de seguridad laboral). Además, los empleados no tienen claridad en la implementación de estrategias para la gestión del cambio al diseño 0 en el desarrollo de sus servicios, por lo cual no se logra garantizar la conformidad del producto y servicio suministrado.

También se logra evidenciar que, dentro de la organización, los empleados preservan los productos durante la prestación del servicio para asegurar la conformidad con los requisitos y se tiene conocimiento de cuál es el estado de conformidad del servicio de acuerdo a los requisitos establecidos, lo que asegura que al momento de presentarse cambios en el diseño o desarrollo de los servicios, los empleados garanticen la conformidad del producto y servicio suministrado. A pesar de esto, se evidencia la necesidad de fortalecer estos temas de preparación y control ante eventos de emergencias, para garantizar el desempeño de las actividades en la organización y dar mayor control a los riesgos asociados a la prestación del servicio.

Por otra parte, cuando se presentan cambios en la prestación del servicio, los empleados están asegurando la conformidad con los requisitos, sin embargo, cuando se generan cambios en el diseño y desarrollo de los servicios, los empleados no lo identifican ni lo controlan de manera adecuada, por eso es necesario sentar bases más sólidas en el control de cambios que se puedan generar en cada uno de los procesos.

En cuanto a la alta dirección, reflejan que sí conocen los tipos de riesgos asociados a la funcionalidad de la organización, pero mencionan que es necesario analizarlos con más frecuencia, esto crea un nivel de incertidumbre ya puede que se tengan bien identificados los riesgos, pero al no ser evaluados constantemente, 
no se están gestionando las medidas necesarias para controlarlos. Igualmente, consideran que el control de riesgos de la organización está asegurando los procesos, pero no existen documentos donde se soporten los controles implementados, y al existir una base documental la estabilidad del negocio puede verse afectada, por lo cual se convierte en un aspecto a reforzar.

En la etapa de planificación, se hace evidente que las medidas de control a riesgos no son implementadas de manera efectiva, y a pesar de expresar que existen acciones de tratamiento y controles eficaces en la gestión del riesgo, es necesario mejorarlos con acciones que ayuden a tratar adecuadamente los riesgos asociados a la prestación del servicio.

Para el SGI es necesario identificar, analizar, valorar y tratar los riesgos en cada uno de los procesos del sistema, los cuales deben contemplar acciones de tratamiento y controles eficaces, razón por la cual es necesario considerarlo dentro de la guía para darle un carácter preventivo y proactivo a los procesos.

En el enfoque de la documentación, los resultados muestran que los procesos se encuentran documentados y que se lleva un buen control en la información documentada por medio de manuales, guías y procedimientos, lo que refleja una buena organización de la información manejada de manera interna.

Adicionalmente, se evidencia que la organización cuenta con un procedimiento definido para la contratación externa, donde determinan los métodos que se deben aplicar a los procesos, productos y servicios contratados externamente, sin embargo, los empleados no reconocen bien la aplicación de esos métodos en la contratación de servicios externos, ya que el manejo de este proceso solo es desempeñado por el área de suministro y no están involucrando a las demás áreas de desempeño de la organización, lo que hace que el manejo de la documentación sea un poco insuficiente.
Por su parte, la alta gerencia considera que mediante la documentación, la organización contribuye a la gestión del conocimiento organizacional y que los manuales, las guías, los procedimientos y los protocolos que se encuentran documentados, establecen de manera efectiva las prácticas de aplicación común de toda la organización; mencionando también que los objetivos de los procedimientos se encuentran acordes y responden de manera efectiva al control de los procesos.

En términos generales, la organización tiene manejo y control de la documentación con prácticas de aplicación que se encuentran acordes a los procesos, lo que facilitará (como un medio importante) la ejecución del SGI.

Dentro de los resultados obtenidos para el enfoque por competencia, se logra identificar que los empleados conocen los niveles de autoridad y las responsabilidades definidas en el SGC, y reconocen que la empresa determina las competencias de las personas que realizan una labor dentro de la organización. Adicionalmente, todos los empleados tienen conocimiento sobre la importancia de las actividades y de cómo contribuyen al logro de los objetivos de la calidad. Por su parte, la alta gerencia considera que la gestión de las competencias de los empleados y los contratistas es un aspecto fundamental dentro de la organización, sin embargo, es evidente asegurar la competencia del personal para que los procesos sean más eficaces, es un aspecto fundamental dentro de la organización.

También se logra identificar que las competencias específicas de cada cargo son evaluadas anualmente, con el fin de hacer seguimiento a las funciones y responsabilidades de los empleados en los procesos en la organización.

Por otra parte, la organización asegura que los líderes de procesos reúnan el conocimiento y la experiencia necesaria para llevar a cabo todas las funciones y satisfacer las necesidades de los clientes y demás partes 
interesadas. Además, se realizan las gestiones correspondientes para ejecutar los procesos adecuadamente y consideran que las competencias se aseguran, se mantienen y se mejoran mediante la gestión del talento humano.

En términos generales, la organización asegura la formación y experiencia de sus empleados, teniendo en cuenta las aptitudes que tiene el personal frente al desempeño de sus funciones, para garantizar una buena gestión del talento humano dentro de la organización.

En el enfoque de seguimiento y mejora, los resultados reflejan que en la organización se logra identificar y controlar las salidas no conformes para prevenir su uso o entrega al cliente. Adicionalmente, la organización evalúa el desempeño y la eficacia del SGC a partir de los resultados del seguimiento y la medición de los procesos, productos y servicios.

Los empleados realizan seguimiento a la percepción de sus clientes en la prestación del servicio, reflejan conocimiento de las auditorías internas y externas realizadas al SGC de la organización y consideran que la gerencia revisa el SGC para asegurar su conveniencia, adecuación, eficacia y alineación con la planificación estratégica. Adicionalmente, reconocen que dentro de la empresa existen mecanismos para seleccionar las oportunidades de mejora que contribuyen a aumentar la satisfacción de los clientes.

Por su parte, la alta gerencia cree que los procesos de la organización se están evaluando de manera efectiva y que los procesos actuales cumplen los objetivos de la empresa, y no consideran que el seguimiento, la medición y el mejoramiento sean aspectos débiles dentro de su organización, ya que se realiza seguimiento y mejoramiento del SGC mediante auditorías internas.

Adicionalmente, los mecanismos de medición y seguimiento se encuentran bien definidos y acordes a las necesidades de la organización. De igual forma, la organización dispone de la información necesaria para tomar decisiones que logren corregir los incumplimientos, prevenir riesgos y mejorar los procesos.

Así mismo, considera que dentro de la organización se realizan reuniones donde se analizan los resultados y se toman decisiones, sin embargo, se tiene que mejorar la disponibilidad en la entrega de la información, ya que puede llegar a afectar la conformidad de los procesos por no definir los criterios de manera oportuna.

En términos generales, la organización cuenta con mecanismos de medición y seguimiento a los procesos, pero se debe reforzar la disponibilidad de la información y la gestión eficaz de los incumplimientos que se generan en las auditorías realizadas.

Por último, para el enfoque de liderazgo, se logra identificar que la mayoría de los empleados consideran que la gerencia muestra liderazgo y compromiso hacia el SGC y que también planifica los cambios en el sistema e identifica sus consecuencias, sin embargo, los empleados no cuentan con los conocimientos suficientes para enfrentar los cambios del sistema de gestión, temas que deben ser intervenidos.

Por su parte, los empleados consideran que la gerencia no determina y ni proporciona los recursos necesarios para el establecimiento, implementación, mantenimiento y mejora continua del SGC. Tampoco determinan ni proporcionan el personal, la infraestructura, el ambiente y los recursos necesarios para implementar de manera eficaz el SGC y lograr la conformidad de los productos y servicios, pero síreconocen que dentro de la organización se gestionan los conocimientos necesarios para la operación de los procesos y el logro en la conformidad de los productos y servicios.

Estos resultados hacen evidente la necesidad de mejorar los aspectos de liderazgo como la planeación de los 
recursos, personal y espacios de trabajo por parte de la alta dirección, para lograr una prestación del servicio de forma integral, donde los empleados tengan todos los elementos necesarios para desempeñar sus funciones y donde se sientan acompañados y respaldados por sus superiores en la ejecución de los procesos.

\section{Guía de implementación del sistema de gestión integral}

Teniendo en cuenta el diagnóstico actual de la organización, los enfoques establecidos y analizados dan un punto de partida para la elaboración de la guía de gestión integral. Las consideraciones de los requisitos de la norma evaluados evidencian las necesidades de la organización en los temas de gestión.

El documento guía para la implementación de un SGI, es el resultado más relevante dentro de la investigación y se encuentra diseñado de la siguiente forma:

1. Objetivos: plantean el propósito de la guía, entre los cuales se contempla ajustarla a las necesidades de la organización, definir los parámetros de implementación y la comunicación de esta misma con todas las dependencias de la organización.

2. Ámbito de aplicación: la guía especifica los requisitos para un sistema de gestión integral en la organización.

3. Siglas o abreviaciones: se especifican algunas siglas, relacionadas con sistemas de gestión.

4. Definiciones generales: con el fin de facilitar la comprensión de la guía, se definen algunos términos.

5. Carácter de integralidad del sistema de gestión: en este aparte se justifica la intención de dar el enfoque integral a la guía, teniendo como teoría base el libro Sistema de gestión integral. Una sola gestión, un solo equipo.
6. Principios de la gestión integral: la guía para el SGI propuesto se encuentra alineado bajo los principios de gestión por procesos, partes interesadas, cumplimiento normativo, gestión de riesgos, gestión de la documentación, competencia, medición, análisis y mejora, basado en el libro Sistema de gestión integral. Una sola gestión, un solo equipo (Atehortúa, Bustamante y Valencia, 2008, p. 47) y las variables incluidas del contexto integral y liderazgo.

7. Los pasos de implementación: en este apartado se define paso a paso la implementación de la guía, teniendo en cuenta la estructura de alto nivel de las últimas versiones de las normas ISO 9001 e ISO 14001, el cual se encuentra estructurado de la siguiente forma:

\section{Contexto de la organización}

\section{Comprensión de la organización y su contexto.}

En este numeral se realizan recomendaciones que involucran los siguientes aspectos:

- Contexto externo: análisis del negocio a nivel nacional, regional y local: para el desarrollo de este análisis se propone a la organización, tener en cuenta el posicionamiento del sector en el cual se mueve desde el ámbito nacional, regional y local, para lo cual es necesario conocer el comportamiento del sector de servicios públicos domiciliarios, comportamiento de clientes, competidores, etc.

- Contexto político, económico, legal, social, tecnológico y ambiental: es necesario considerar estos aspectos para poder ubicar el nivel en el cual se encuentra la organización y poder proyectar su crecimiento. Con este análisis se logran identificar las amenazas y fortalezas a las que puede enfrentar la organización.

- Contexto interno: estrategias corporativas, procesos internos, estructura organizacional y capacidad 
tecnológica con la que cuenta la organización: estos aspectos se deberían considerar para identificar la capacidad competitiva, las fortalezas y las debilidades de la organización.

\section{Comprensión de las expectativas y necesidades de las partes interesadas.}

Cada empleado debería tener claridad de las expectativas y necesidades de las partes interesadas implicadas en la prestación del servicio. Entre las partes interesadas más relevantes de la organización, se relacionan los clientes (suscriptores del servicio de acueducto), proveedores, autoridades, empleados de la organización y gremios del sector de servicios públicos, adicionalmente, se debe considerar como partes interesadas el medio ambiente físico, biótico y social (desde el enfoque de la ISO 14001:2015).

\section{Determinación del alcance del sistema de gestión.}

La organización debería definir su alcance teniendo en cuenta los movimientos del sector y las implicaciones sociales, culturales, políticas y ambientales, en las cuales se ven involucradas las unidades de servicio del negocio. Adicionalmente, deberían tener en cuenta otros criterios para definir el alcance tales como: la determinación de los requisitos de las partes interesadas y los requisitos de los productos y servicios, tomando como punto de partida el SGG ya implementado.

\section{Sistema de gestión y sus procesos.}

La propuesta del SGI va enfocada a integrar la gestión dentro de la organización, para lo cual se debería trabajar de la mano con los empleados, involucrando todas las dependencias de la organización en los temas de interés para la prestación del servicio de acueducto, con el fin de fortalecer la comprensión y el conocimiento del ámbito en el que opera la organización.

\section{Liderazgo}

\section{Liderazgo y compromiso.}

La gerencia y los líderes de cada proceso deberían orientar y direccionar el rumbo de la organización, con miras a dar cumplimiento a sus objetivos estratégicos. Para realizarlo y comprometer a todos los niveles de la organización, es necesario implementar las siguientes acciones:

- Definir y alinear la gestión integral estratégica de la organización, en temas relacionados con la misión, visión, política, objetivos y las estrategias de intervención.

- Empoderar a todos los empleados en la consecución de los objetivos estratégicos.

- Dirigir, apoyar y comprometer a los empleados en la gestión eficaz de los procesos definidos.

- Planificar, proporcionar y garantizar que todos los elementos, recursos, infraestructura y ambientes laborales, se ejecuten de manera segura.

- Orientar a los empleados en cómo comprender e implementar los cambios que trae consigo el SGI.

- Promover el trabajo en equipo entre los empleados y empoderarlos en la toma de iniciativas para la resolución de problemas.

\section{Politica.}

Se propone a la organización establecer, implementar y mantener una política integral que recoja los propósitos de la organización, contemplando los requisitos de calidad, ambiente y de SST.

\section{Roles, autoridades y responsabilidades en la organización.}

Para consolidar la gestión integral dentro de la organización, las directivas deberían asignar los roles y 
responsabilidades de cada empleado, y velar por que se cumplan de manera eficaz y eficiente.

\section{Participación y consulta.}

La organización debería considerar en todo momento la participación de los trabajadores en temas de SST, tales como la identificación de peligros, valoración de riesgos, investigación de accidentes y consulta de los mecanismos de participación que la organización emplee.

De igual forma, se debería considerar la participación y consulta de trabajadores en los temas de valoración de aspectos e impactos ambientales asociados a las actividades desempeñadas.

\section{Planificación}

\section{Planificación de acciones en el sistema de gestión integral.}

Para la planificación de las acciones se tienen en cuenta algunos criterios expuestos en el libro SGI. Una sola gestión, un solo equipo (Atehortúa, Bustamante y Valencia, 2008, p. 77). Dentro de la guía de gestión integral, se propone implementar los procesos, teniendo en cuenta las entradas, acciones y salidas propuestas. A continuación, se detallan los objetivos de cada uno de ellos:

\section{Procesos estratégicos.}

Proceso de gestión estratégica.

Objetivo: guiar a la organización en la consecución de su visión, por medio de instrumentos que orienten la gestión de los procesos del SGI.

\section{Proceso de gestión de talento humano.}

Objetivo: aumentar la competencia de las personas y lograr un desarrollo integral de los trabajadores.

\section{Procesos misionales.}

Proceso de gestión de los clientes.

Objetivo: identificar las necesidades de los clientes y fijar los canales de comunicación con ellos, para facilitar acuerdos y cumplir con sus requerimientos.

\section{Proceso de gestión de la prestación del servicio.}

Objetivo: ofrecer un producto y servicio que cumpla con todos los estándares de calidad, evitando el menor impacto al ambiente y asegurando la integridad de los trabajadores.

\section{Procesos de apoyo.}

Procesos de gestión financiera.

Objetivo: dirigir e integrar las actividades de planeación financiera, la ejecución presupuestal, el control de recursos financieros, registro de los movimientos financieros y presentación de informes.

\section{Proceso de gestión ambientaly SST.}

Objetivo: controlar los impactos que se derivan de la interacción entre la organización y su entorno físico, y las relaciones de los empleados y los aspectos que pueden afectar su seguridad y salud.

\section{Proceso de gestión de los recursos físicos y tecnológicos.}

Objetivo: suministrar, mantener y mejorar los materiales y la infraestructura básica para desarrollar las actividades adecuadamente.

\section{Proceso gestión de la información.}

Objetivo: tener la información disponible y asegurar la confidencialidad e integridad de la esta misma para aportar a la gestión del conocimiento organizacional. 
Tabla 3. Entradas, acciones y salidas del proceso de gestión estratégica

\begin{tabular}{|l|l|l|}
\hline \multicolumn{1}{|c|}{ Entradas } & \multicolumn{1}{c|}{ Acciones } & \multicolumn{1}{c|}{ Salidas } \\
\hline Marco normativo & Gestión de la normativa aplicable & Procedimiento de gestión normativa \\
\hline Marco normativo & Determinación de la plataforma estratégica & Matriz normativa \\
\hline Necesidades y expectativas de los clientes & $\begin{array}{l}\text { Determinación de la plataforma axiológica y } \\
\text { deontológica }\end{array}$ & $\begin{array}{l}\text { Plataforma estratégica (misión, visión, plan } \\
\text { estratégico) }\end{array}$ \\
\hline $\begin{array}{l}\text { Necesidades y expectativas de otras partes } \\
\text { interesadas }\end{array}$ & Determinación del modelo de procesos & Código de ética \\
\hline Información del entorno & Determinación de la estructura organizacional & Código de políticas de gestión \\
\hline & Gestión de los riesgos & Modelo de procesos \\
\hline & Gestión de la comunicación organizacional & Estructura organizacional \\
\hline & & Matriz de riesgos \\
\hline
\end{tabular}

Fuente: Atehortúa, Bustamante y Valencia, Sistema de Gestión Integral. Una sola gestión, un solo equipo, p. 80.

Tabla 4. Entradas, acciones y salidas del proceso de gestión de talento humano

\begin{tabular}{|l|l|l|}
\hline \multicolumn{1}{|c|}{ Entradas } & \multicolumn{1}{c|}{ Acciones } & \multicolumn{1}{c|}{ Salidas } \\
\hline Matriz normativa & $\begin{array}{l}\text { Planificación de la satisfacción de necesidades } \\
\text { en gestión humana }\end{array}$ & $\begin{array}{l}\text { Talento humano con la competencia } \\
\text { requerida y consciente de la importancia de su } \\
\text { trabajo frente al SGI }\end{array}$ \\
\hline $\begin{array}{l}\text { Plataforma estratégica de la entidad (misión, } \\
\text { visión y plan estratégico) }\end{array}$ & Selección del talento humano & Programas de bienestar laboral \\
\hline Política de gestión humana & Inducción del talento humano & Planes de mejoramiento individual \\
\hline Necesidades en gestión humana & Administración del talento humano & \\
\hline Ofertas de programas de gestión humana & Formación del talento humano & \\
\hline Estructura organizacional definida & Gestión del bienestar laboral & \\
\hline Indicadores de desempeño & Evaluación del desempeño & \\
\hline & Mejoramiento del desempeño & \\
\hline
\end{tabular}

Fuente: Atehortúa, Bustamante y Valencia, Sistema de Gestión Integral. Una sola gestión, un solo equipo, p. 155.

Tabla 5. Entradas, acciones y salidas del proceso de gestión de los clientes

\begin{tabular}{|l|l|l|}
\hline \multicolumn{1}{|c|}{ Entradas } & \multicolumn{1}{|c|}{ Acciones } & \multicolumn{1}{c|}{ Salidas } \\
\hline Marco normativo & Identificar las necesidades de los clientes & $\begin{array}{l}\text { Reconocimiento de las necesidades de los } \\
\text { clientes }\end{array}$ \\
\hline $\begin{array}{l}\text { Plataforma axiológica (código de ética) y } \\
\text { deontológica (políticas de gestión) }\end{array}$ & $\begin{array}{l}\text { Establecer acuerdos para la elaboración y } \\
\text { prestación del servicio }\end{array}$ & $\begin{array}{l}\text { Acuerdo sobre los requerimientos del } \\
\text { producto o servicio }\end{array}$ \\
\hline $\begin{array}{l}\text { Plataforma estratégica de la entidad (misión, } \\
\text { visión y plan estratégico) }\end{array}$ & $\begin{array}{l}\text { Establecer mecanismos de comunicación con } \\
\text { los clientes }\end{array}$ & Mecanismos de comunicación con los clientes \\
\hline $\begin{array}{l}\text { Información acerca del público objetivo del } \\
\text { producto y servicio (mercado potencial) }\end{array}$ & Atender la realimentación de los clientes & Atención a la realimentación de los clientes \\
\hline & Evaluar la satisfacción de los clientes & $\begin{array}{l}\text { Información sobre la satisfacción de los } \\
\text { clientes }\end{array}$ \\
\hline
\end{tabular}

Fuente: Atehortúa, Bustamante y Valencia, Sistema de Gestión Integral. Una sola gestión, un solo equipo, p. 110. 
Tabla 6. Entradas, acciones y salidas del proceso de gestión de la prestación del servicio

\begin{tabular}{|l|l|l|}
\hline \multicolumn{1}{|c|}{ Entradas } & \multicolumn{1}{|c|}{ Acciones } & \multicolumn{1}{c|}{ Salidas } \\
\hline Marco normativo & $\begin{array}{l}\text { Establecer los requisitos del producto y } \\
\text { servicio }\end{array}$ & Requisitos del producto y servicio \\
\hline $\begin{array}{l}\text { Plataforma estratégica de la entidad (misión, } \\
\text { visión y plan estratégico) }\end{array}$ & $\begin{array}{l}\text { Planeación de la realización del producto y } \\
\text { servicio }\end{array}$ & Plan de producción y prestación del servicio \\
\hline $\begin{array}{l}\text { Plataforma axiológica (código de ética) y } \\
\text { deontológica (políticas de gestión) }\end{array}$ & Diseño del producto y servicio & Diseño del producto y servicio \\
\hline $\begin{array}{l}\text { Recursos humanos, financieros, físicos, } \\
\text { tecnológicos y de información }\end{array}$ & $\begin{array}{l}\text { Producción del producto y prestación del } \\
\text { servicio }\end{array}$ & Producto y servicio \\
\hline $\begin{array}{l}\text { Acuerdos sobre los requerimientos del } \\
\text { producto y servicio (requisitos) }\end{array}$ & & \\
\hline
\end{tabular}

Fuente: Atehortúa, Bustamante y Valencia, Sistema de Gestión Integral. Una sola gestión, un solo equipo, p. 118.

Tabla 7. Entradas, acciones y salidas del proceso de gestión financiera

\begin{tabular}{|l|l|l|}
\hline \multicolumn{1}{|c|}{ Entradas } & \multicolumn{1}{|c|}{ Acciones } & \multicolumn{1}{c|}{ Salidas } \\
\hline Marco normativo & Planeación financiera & $\begin{array}{l}\text { - Políticas financieras institucionales } \\
\text { Proyecciones financieras, ingresos, } \\
\text { inversión y gastos } \\
\text { Presupuesto de la organización }\end{array}$ \\
\hline $\begin{array}{l}\text { Plataforma estratégica de la entidad (misión, } \\
\text { visión y plan estratégico) }\end{array}$ & Ejecución y control de los recursos financieros & $\begin{array}{l}\text { Certificados de reserva y disponibilidad } \\
\text { presupuestal } \\
\text { Pago de obligaciones } \\
\text { Recaudo de ingresos }\end{array}$ \\
\hline $\begin{array}{l}\text { Plataforma axiológica (código de ética) y } \\
\text { deontológica (políticas de gestión) }\end{array}$ & $\begin{array}{l}\text { Requisitos de movimientos contables y } \\
\text { elaboración de informes financieros. }\end{array}$ & $\begin{array}{l}\text { - Informe de estados financieros de } \\
\text { propósito general } \\
\text { Informe de estados financieros }\end{array}$ \\
\hline $\begin{array}{l}\text { Proyección de necesidades de recursos } \\
\text { financieros (inversión y egresos) de los } \\
\text { procesos }\end{array}$ & & \begin{tabular}{l} 
\\
\hline Proyección de ingresos
\end{tabular} \\
\hline
\end{tabular}

Fuente: Atehortúa, Bustamante y Valencia, Sistema de Gestión Integral. Una sola gestión, un solo equipo, p. 134.

Tabla 8. Entradas, acciones y salidas del proceso de gestión ambiental y SST

\begin{tabular}{|l|l|l|}
\hline \multicolumn{1}{|c|}{ Entradas } & \multicolumn{1}{|c|}{ Acciones } & \multicolumn{1}{c|}{ Salidas } \\
\hline Marco normativo & $\begin{array}{l}\text { Identificación de los aspectos ambientales } \\
\text { significativos y de los peligros y riesgos } \\
\text { laborales }\end{array}$ & $\begin{array}{l}\text { Procedimientos para la identificación, análisis } \\
\text { y valoración de los aspectos ambientales } \\
\text { significativos y de los peligros y riesgos } \\
\text { laborales }\end{array}$ \\
\hline $\begin{array}{l}\text { Políticas de gestión ambiental y de seguridad } \\
\text { y salud laboral }\end{array}$ & $\begin{array}{l}\text { Definición de los programas de gestión } \\
\text { ambiental y de seguridad y salud laboral }\end{array}$ & $\begin{array}{l}\text { Matriz de aspectos e impactos ambientales } \\
\text { - Matriz de peligros y riesgos laborales }\end{array}$ \\
\hline Plan estratégico & $\begin{array}{l}\text { Establecimiento de los controles operacionales } \\
\text { aplicables }\end{array}$ & $\begin{array}{l}\text { Programa de gestión ambiental y de seguridad } \\
\text { y salud en el trabajo }\end{array}$ \\
\hline Formato de la matriz de riesgos & $\begin{array}{l}\text { Establecimiento de los procedimientos de } \\
\text { preparación y respuesta ante emergencias }\end{array}$ & $\begin{array}{l}\text { Procedimientos de control operacional } \\
\text { Procedimientos de preparación y respuesta } \\
\text { ante emergencias }\end{array}$ \\
\hline
\end{tabular}

Fuente: Atehortúa, Bustamante y Valencia, Sistema de Gestión Integral. Una sola gestión, un solo equipo, p. 144. 
Tabla 9. Entradas, acciones y salidas del proceso de gestión de los recursos físicos y tecnológicos

\begin{tabular}{|l|l|l|}
\hline \multicolumn{1}{|c|}{ Entradas } & \multicolumn{1}{|c|}{ Acciones } & \multicolumn{1}{c|}{ Salidas } \\
\hline Matriz normativa & $\begin{array}{l}\text { Definir los requisitos de los recursos físicos y } \\
\text { tecnológicos }\end{array}$ & $\begin{array}{l}\text { Información de los requisitos de los recursos } \\
\text { físicos y tecnológicos }\end{array}$ \\
\hline $\begin{array}{l}\text { Plataforma estratégica de la entidad (misión, } \\
\text { visión y plan estratégico) }\end{array}$ & Provisión de recursos físicos y tecnológicos & $\begin{array}{l}\text { Recursos físicos y tecnológicos } \\
\text { Grupo de proveedores seleccionados y } \\
\text { evaluados }\end{array}$ \\
\hline $\begin{array}{l}\text { Plataforma axiológica (código de ética) y } \\
\text { deontológica (políticas de gestión) }\end{array}$ & $\begin{array}{l}\text { Efectuar mantenimiento a los recursos físicos } \\
\text { y tecnológicos }\end{array}$ & $\begin{array}{l}\text { Recursos físicos y tecnológicos disponibles } \\
\text { para su uso y operación }\end{array}$ \\
\hline $\begin{array}{l}\text { Necesidades de los recursos físicos y } \\
\text { tecnológicos de los procesos }\end{array}$ & Controlar los equipos de medición & Equipos de medición \\
\hline Presupuesto de la entidad & & \\
\hline
\end{tabular}

Fuente: Atehortúa, Bustamante y Valencia, Sistema de Gestión Integral. Una sola gestión, un solo equipo, p. 16.

Tabla 10. Entradas, acciones y salidas del proceso de gestión de la información

\begin{tabular}{|l|l|l|}
\hline \multicolumn{1}{|c|}{ Entradas } & \multicolumn{1}{c|}{ Acciones } & \multicolumn{1}{c|}{ Salidas } \\
\hline Matriz normativa & Identificación de la información & Información \\
\hline $\begin{array}{l}\text { Plataforma axiológica (código de ética) y } \\
\text { deontológica (políticas de gestión) }\end{array}$ & Captura de la información & $\begin{array}{l}\text { Procedimientos del proceso de gestión de la } \\
\text { información }\end{array}$ \\
\hline Políticas de gestión de la información & Organización de la información & Matriz de riesgo de seguridad de la información \\
\hline Necesidades en la gestión de la información & Distribución de la organización & \\
\hline $\begin{array}{l}\text { Oferta de productos y servicios en gestión de } \\
\text { la información }\end{array}$ & Utilización de la información & \\
\hline Formato de matriz de riesgo & $\begin{array}{l}\text { Gestión de los riesgos relacionados con la } \\
\text { seguridad de la información }\end{array}$ & \\
\hline
\end{tabular}

Fuente: Atehortúa, Bustamante y Valencia, Sistema de Gestión Integral. Una sola gestión, un solo equipo, p. 184.

Tabla 11. Entradas, acciones y salidas del proceso de gestión de la evaluación y el mejoramiento

\begin{tabular}{|l|l|l|}
\hline \multicolumn{1}{|c|}{ Entradas } & \multicolumn{1}{c|}{ Acciones } & \multicolumn{1}{c|}{ Salidas } \\
\hline Gestión normativa & Autoevaluación del SGI & Informes de rendición de cuentas \\
\hline Matriz normativa & Evaluación independiente del SGI & Planes de mejoramiento por procesos \\
\hline $\begin{array}{l}\text { Plataforma estratégica de la entidad (misión, } \\
\text { visión y plan estratégico) }\end{array}$ & $\begin{array}{l}\text { Análisis de los resultados de la evaluación } \\
\text { del SGI }\end{array}$ & Plan de mejora organizacional \\
\hline $\begin{array}{l}\text { Plataforma axiológica (código de ética) y } \\
\text { deontológica (políticas de gestión) }\end{array}$ & Análisis de las causas de incumplimiento & \\
\hline Modelo de procesos & $\begin{array}{l}\text { Definición de los planes de mejora por } \\
\text { procesos }\end{array}$ & \\
\hline Estructura organizacional & Revisión del SGI por la dirección & \\
\hline Mapa de riesgos & $\begin{array}{l}\text { Definición del plan de mejoramiento } \\
\text { organizacional }\end{array}$ & \\
\hline Matriz de comunicaciones & & \\
\hline $\begin{array}{l}\text { Necesidades y expectativas de los clientes y } \\
\text { otras partes integradas }\end{array}$ & & \\
\hline Información del entorno & & \\
\hline
\end{tabular}

Fuente: Atehortúa, Bustamante y Valencia, Sistema de Gestión Integral. Una sola gestión, un solo equipo, p. 194. 


\section{Procesos de evaluación.}

Proceso de gestión de la evaluación y el mejoramiento.

Objetivo: verificar y ajustar todo el sistema para identificar lo que se hizo y se logró, e indicar a la dirección la necesidad de emprender acciones de mejora.

\section{Apoyo}

\section{Recursos.}

Generalidades.

Los procesos de apoyo definidos deberían garantizar y proporcionar los recursos necesarios para el establecimiento, implementación, mantenimiento y mejora continua del SGI, para esto se requiere indispensablemente el compromiso por parte de la dirección.

\section{Personas.}

En dichos procesos se contempla la gestión del talento humano, los cuales pueden garantizar las personas necesarias y competentes para implementar de manera eficaz el SGI. Se debería considerar y gestionar las competencias de las personas.

\section{Infraestructura y ambiente para la operación de los procesos.}

Se puede considerar todos los aspectos relacionados a proporcionar una infraestructura y un ambiente óptimo para el desarrollo eficaz de los procesos y la conformidad de productos y servicios suministrados.

\section{Recursos de seguimiento y medición.}

Se debería tener apoyo de técnicas metrológicas que garanticen la confiabilidad y validez de los datos tomados. Adicionalmente, se puede considerar el proceso de gestión de recursos físicos y tecnológicos definido en el numeral de planificación.

\section{Gestión del conocimiento.}

Para dar una gestión integral óptima, la organización debería suministrar todo el conocimiento necesario para desempeñar adecuadamente el SGI, para lo cual es necesario facilitar y fortalecer los canales de comunicación interna y externa que tiene la organización. Esta gestión promueve la eficiencia en la toma de decisiones. La organización debería determinar cuál es el conocimiento necesario para llevar a cabo cada uno de los procesos, mantener y poner a disposición ese conocimiento, igualmente poder adquirir 0 acceder a nuevos conocimientos.

\section{Toma de conciencia.}

Es responsabilidad de los empleados tomar conciencia de la política integral y de los objetivos de la organización, además de conocer e interiorizar los aportes que le hacen desde su labor y las implicaciones de un incumplimiento a los requisitos del SGI. Es necesario que la organización incentive y motive a los empleados a tomar conciencia mediante capacitaciones, socializaciones, prácticas laborales y actividades interactivas que generen la toma de conciencia en aspectos de manejo de la calidad, ambiente y seguridad.

\section{Información documentada.}

Se encuentra apoyada bajo el proceso de gestión de la información.

\section{Operación}

\section{Planificación y control operacional.}

Todos los procesos planificados deberían ejecutarse con todas las medidas de control operacional establecidas y cumplir con las actividades estipuladas en cada 
proceso. Igualmente, es necesario tener en cuenta las acciones de control a implementar en caso de generarse cambios en la planificación.

\section{Requisitos para los productos y servicios.}

Para determinar los requisitos de los productos y servicios, se sugiere inicialmente realizar una investigación del mercado involucrado en la prestación de servicio domiciliario y del producto entregado (agua potable), consultar informes de servicios e indagar sobre nuevas tecnologías. Una vez realizado, es importante clasificar la información teniendo en cuenta tanto las exigencias legales asociadas, como las reglamentarias aplicables y las que considere la organización; al finalizar, se sugiere sacar un listado de todos los requisitos identificados.

\section{Diseño y desarrollo de los productos y servicios.}

La organización puede considerar procesos de diseño y desarrollo en caso de requerirse, para asegurar y preservar el producto y servicio suministrado, para lo cual es necesario contemplar su planificación, requisitos, controles y cambios.

\section{Control de los procesos, productos y servicios adquiridos y suministrados externamente.}

La organización puede asegurar que los procesos, productos y servicios suministrados externamente estén conforme a los requisitos, para lo cual es necesario contemplar tipo, alcance y manejo de información con proveedores externos.

Se deberían ejercer controles a todos los procesos de compra, se sugiere a la organización mejorar el control de inventario para poder hacer más eficaz la gestión de recursos. Adicionalmente, se debería contar con información de proveedores actualizada y realizar una evaluación y análisis de las cotizaciones de productos y servicios antes de adquirirlos.

\section{Producción y provisión del servicio.}

La organización debería implementar la producción y provisión del servicio de acueducto bajo condiciones controladas. Puede considerar el capítulo 8.5 de la norma ISO 9001:2015.

\section{Gestión del cambio.}

Teniendo en cuenta los requisitos de las normas involucradas (ISO 9001, ISO 14001, OSHAS 18001), se sugiere a la organización asegurar que la información documentada sea modificada cuando se cambien los requisitos para los productos y servicios, se generen cambios en la normativa, en los procesos, en los requisitos de las partes interesadas o en la tecnología. De igual forma, es importante que todas las personas sean conscientes de los requisitos modificados.

\section{Liberación de los productos y servicios.}

Se debería garantizar que la entrega del producto y servicio al cliente pueda hacerse cuando se hayan completado las disposiciones planificadas en los procesos. Es necesario contar con toda la información relacionada a la prestación del servicio de acueducto.

\section{Preparación y respuesta ante emergencias.}

Se debería contar con un plan de emergencia, el cual puede ir acompañado de un programa de formación ante emergencias de tipo laboral y ambiental que permita presentarse en las instalaciones de la organización, incluidas las oficinas ubicadas en cada corregimiento.

\section{Control de las salidas no conformes.}

Se deberían identificar y controlar las salidas no conformes para prevenir su uso o entrega no intencionada. Adicionalmente, es necesario tomar acciones correctivas para evitar su reincidencia y comunicar a los clientes en caso de requerirse. 


\section{Evaluación del desempeño}

\section{Seguimiento y medición.}

La organización debería implementar métodos de seguimiento y medición, por lo cual se sugiere a la organización tener en cuenta inicialmente el contexto de la organización, información de los requisitos de las partes interesadas, procesos, objetivos de SGI, estado de conformidad de los productos y servicios y los procesos con proveedores. La organización se puede apoyar de una lista de chequeo que involucre los temas expuestos anteriormente.

\section{Evaluación y análisis.}

La organización debería analizar y evaluar los datos y la información que surge al realizar el seguimiento y medición de los procesos, donde se incluyan la conformidad de los productos y servicios, satisfacción del cliente, desempeño y la eficacia del SGI, si lo planificado se ha implementado de forma eficaz, efectividad de las acciones tomadas para abordar los riesgos y oportunidades, el desempeño de los proveedores externos y la necesidad de mejoras en el SGI.

\section{Auditoría interna.}

La organización debería llevar a cabo auditorías internas a intervalos planificados para proporcionar información acerca del estado del SGI. Se sugiere tener en cuenta los lineamientos de la NTC 19011 Directrices para la auditoría de los sistemas de gestión de la calidad y ambiental.

\section{Revisión por la dirección.}

La alta dirección debería revisar el SGI de la organización en periodos planificados, para asegurar su conveniencia, adecuación, eficacia y alineación continua con la dirección estratégica de la organización.

\section{Mejora}

\section{No conformidad y acción correctiva.}

Al generarse no conformidades, es necesario tomar acciones correctivas que incluyan análisis de causas, revisiones, seguimientos y evaluación de la eficacia.

\section{Mejora continua.}

La organización debería mejorar continuamente la conveniencia, adecuación y eficacia del SGI. Las necesidades u oportunidades deberín considerarse como parte de la mejora continua.

\section{Mapa de procesos}

Teniendo en cuenta las consideraciones del apartado 7 , se realiza el diseño del mapa de procesos.

El proceso de validación de la guía del SGI, se realiza con dos expertos en sistemas de gestión integral, cabe resaltar que estos expertos son los autores de la teoría de referencia de la presente investigación.

Para analizar la información suministrada por los expertos, se determinaron cinco criterios de validación que aplican para cada uno de los ítems propuestos en el instrumento: coherencia, pertinencia, eficacia, contenido y aplicabilidad. A continuación, se realiza la definición de cada uno de los criterios de validación propuestos para la guía:

Dentro de las observaciones y recomendaciones realizadas por los expertos, se recomienda tener presente las normas legales que aplican al sector de servicio público domiciliario y al contexto de la compañía objeto de estudio, ya que existen requisitos específicos del sector que deben cumplirse, como la implementación de un plan de emergencias específico para organizaciones de servicios públicos o el cumplimiento de los requisitos legales que obligan a los operadores de la 
Figura 1. Mapa de proceso propuesto

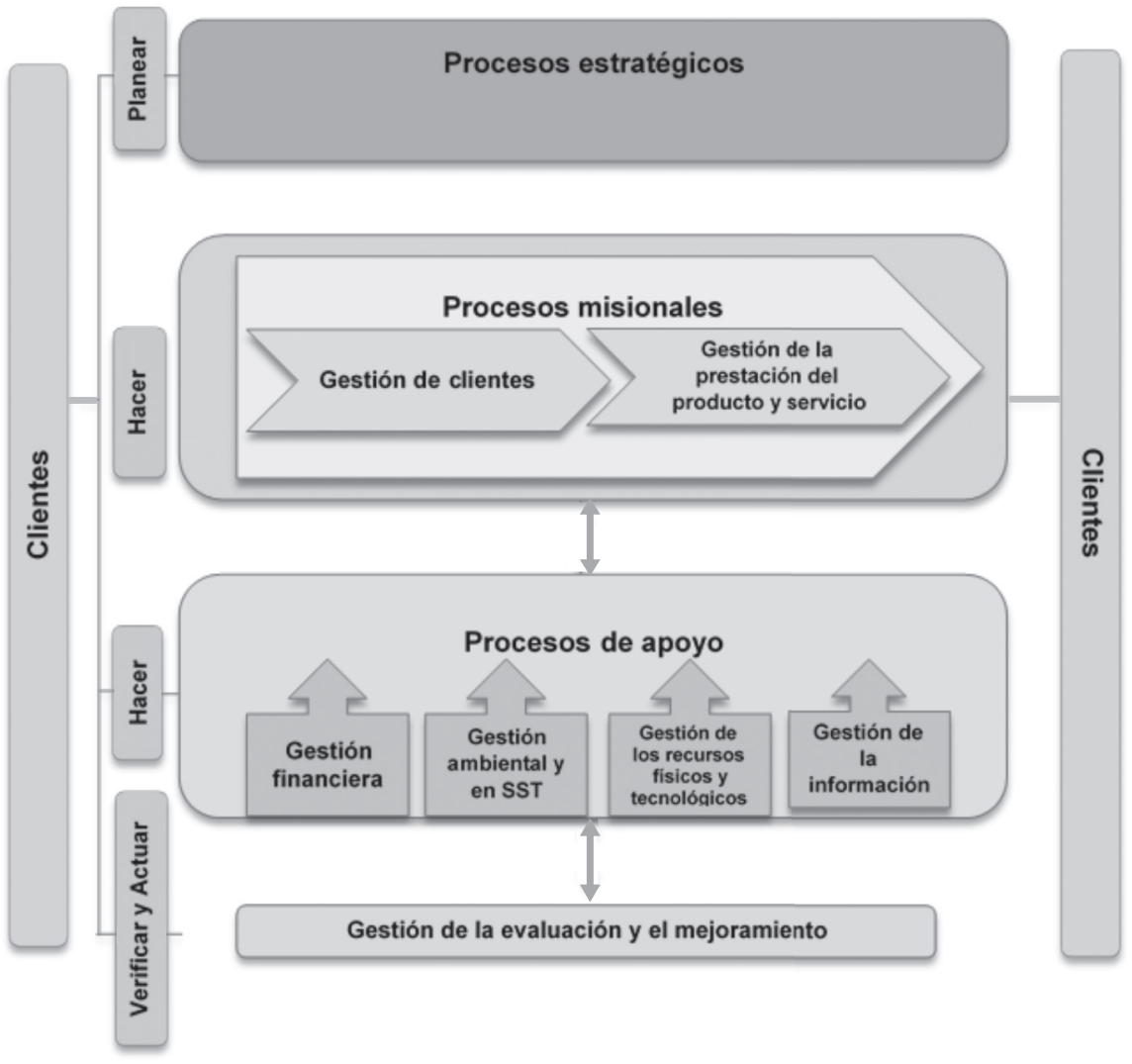

Fuente: elaboración propia basada en el diseño de la guía del SGI.

Tabla 12. Criterios de validación de la guía

\begin{tabular}{|l|l|}
\hline \multicolumn{1}{|c|}{ Criterios de validez } & \multicolumn{1}{c|}{ Definición } \\
\hline Coherencia & $\begin{array}{l}\text { Relación lógica y consecuente entre las necesidades identificadas en el diagnóstico de la organización } \\
\text { y los principios, preceptos y lineamientos definidos en la guía. }\end{array}$ \\
\hline Pertinencia & Es adecuado u oportuno al contexto determinado. \\
\hline Eficacia & La guía responde al objetivo para la cual está construida. \\
\hline Contenido & Si el contenido de la guía es coherente con el marco teórico y conceptual. \\
\hline Aplicabilidad & La guía permite su aplicación en el contexto determinado. \\
\hline
\end{tabular}

Fuente: elaboración propia.

empresa de servicios públicos a estar certificados en competencias laborales.

Por su parte, mencionan que en la política integral es importante considerar el Decreto 1072 de 2015, donde se establece un contenido especifico para la política de gestión de seguridad y salud en el trabajo con requisitos a cumplir. Igualmente, expresan que la guía debería contemplar el nuevo concepto de la ISO 14001:2015 asociado al ciclo de vida y especificar de manera más detallada cómo la empresa puede cumplir con los requisitos de "Gestión del conocimiento" para la norma 
ISO9001:2015, determinando, manteniendo y poniendo a disposición el conocimiento nuevo y el existente.

De acuerdo con las observaciones y recomendaciones de los expertos anteriormente expuestas, se realizan los ajustes correspondientes a la guía del SGI para la empresa Óptima de Urabá, sin embargo, se infiere que una vez realizados dichos cambios, no fue posible realizar una nueva realimentación de la guía por parte de los expertos.

Como quiera que la guía exige su aplicación en la empresa Óptima de Urabá para la verificación de la hipótesis en cuanto a su eficacia y transformación de los comportamientos organizacionales, a partir de la implementación de los postulados propuestos; a pesar de ello, y con el ánimo de no perder la estructura metodológica de la investigación, se pone a prueba la guía al proceso de validación por expertos, que para este caso son los proponentes de la teoría base del SGI tomado como referencia; quienes consideraron, en su revisión inicial, que la guía aún no era eficaz, emitiendo observaciones de mejora que fueron acogidas en su totalidad. A pesar de esto, solo se logra contar con esta primera validación sin poder recibir realimentación de los ajustes realizados por motivos ajenos al investigador.

De acuerdo con los resultados, se corrobora la teoría de referencia de los autores Atehortúa, Bustamante y Valencia (2008) en su libro Sistema de gestión integral. Una sola gestión, un solo equipo, en donde se especifica que para lograr una gestión integral, es necesario integrar la gestión mas no el sistema (p. 66). De esta forma se logra ver la organización como aquel sistema que posee un modelo de procesos, el cual responde eficazmente a los requisitos de las normas involucradas, resaltando que en la implementación de las etapas de la investigación, los aspectos centrales son enfocados en la definición del contexto integral de la organización mediante la ejecución de procesos interrelacionados.
Sin embargo, para lograr resultados eficaces, es necesario tener en cuenta que la implementación de la guía se encuentra sujeta a cambios sociales, económicos, políticos, entre otros; lo que conlleva a la organización imponerse a nuevos retos que logren satisfacer sus propias necesidades y las de las partes interesadas, para así garantizar su sostenibilidad y mejora en el futuro. Esta afirmación es corroborada en la literatura internacional, donde se reconoce que el éxito de las organizaciones, ya sean de producción o de servicios, lucrativas 0 sin fines de lucro, depende de la gestión eficiente y eficaz de sus procesos (Ortiz, 2014).

Así mismo, siguiendo la postura de Urquijo que menciona que con los cambios generados, también adoptan políticas gubernamentales donde sus decisiones y acciones inciden en temas como la salud, la educación, el desarrollo económico, la regulación de las actividades empresariales y en general en todos los temas relacionados con el bienestar del país (Urquijo, 2012), es por esto que la guía de gestión integral proporciona herramientas a la organización para enfrentar las transformaciones generadas en los mercados internacionales por causa de la globalización, las cuales obligan a las organizaciones a generar cambios en su administración, en su infraestructura y en las actividades empresariales en general.

En este contexto, la satisfacción del cliente se visualiza dentro de la guía como un aspecto primordial que propicia la evaluación de la calidad del servicio y la consolidación del proceso de gestión de clientes bajo un esquema integral, la cual se convierte en una razón de peso para autores como Parasuramant, que expresan que este aspecto es una variable estratégica que aporta ventaja competitiva a las empresas, y por lo cual crea la necesidad de mejorar la satisfacción en los servicios (Parasuramant, 1985).

Otro de los temas importantes que acoge la guía son las medidas laborales, que mediante la norma OSHAS 
18001:2007 propone herramientas para gestionar los desafíos a los que se puede enfrentar la organización en temas de accidentalidad, enfermedades profesionales, jornadas de trabajo, ausentismo laboral, sanciones, medidas de compensación a los trabajadores, entre otros, tal como lo respalda Gerard en el manual práctico para la implantación del estándar OHSAS 18001 (Gerard, 2014).

A pesar de que muchos de estos temas se vienen aplicando, algunas de las organizaciones que han implementado sistemas de gestión de manera integrada, han encontrado dificultades en la selección de un modelo que logre cohesionar sus interacciones, por lo que la mayoría de ellas terminan manejando un sistema dividido en partes sin ningún tipo de relación, lo que conlleva al fracaso del sistema de gestión. Es por esto que el enfoque de la guía también se encuentra centrada en el fortalecimiento de las interacciones entre las actividades desempeñadas en los procesos, no obviando que la conformación del sistema integral suministra el norte que debe llevar la organización para lograr resultados eficaces (Morín, 1994).

En busca de facilitar la implementación de los sistemas de gestión, la Organización Internacional de Normalización - ISO, desarrolló el Anexo SL, un marco para un sistema de gestión genérico y la estructura para todas las normas de sistemas de gestión nuevas y revisadas de ahora en adelante (BSI, 2015). En este sentido, la guía de gestión integral se construye bajo la estructura de alto nivel del Anexo SL, ya que trae como beneficios la disminución en el tiempo de planificación y reducción recursos en su etapa de implementación, En este contexto, Álvarez Forbes respalda que la estructura de alto nivel del anexo SL, posee un enfoque común para los sistemas de gestión, el cual incrementa el valor de las normas para los usuarios, sean estos normalizadores, implementadores, empresas o auditores (Álvarez, 2014).

Como indica Savino (2015), en este sentido, la aplicación de modelos de gestión que abarca los temas de calidad, medio ambiente, salud y seguridad en el trabajo, se han convertido en una actividad importante en la mejora del rendimiento de las empresas y el diseño de los lugares de trabajo más seguros y saludables, sin embargo, a pesar de esta información, aún se logra identificar deficiencias en la implementación de estos modelos de gestión dentro de la organización objeto de estudio, los cuales están relacionados principalmente con el mal concepto de ver que el sistema de gestión solo aduce al cumplimiento de una serie de requisitos exigidos por las normas. Es por esto que la guía busca generar un cambio conceptual en la forma de gestionar sus procesos dentro de la organización, de manera que no solo se cumpla con los requisitos, sino también en la forma de mejorar cada vez más sus indicadores y ser más competitiva y sostenible en el tiempo.

\section{CONCLUSIONES}

Se logra concluir que la implementación del sistema de gestión de calidad (SGC) en la empresa Óptima de Urabá se encuentra aún en una etapa de fortalecimiento, los temas en manejo ambiental, seguridad y salud en el trabajo (SST) no son incluidos como elementos de cohesión a dicho sistema y algunos de sus procesos requieren mayor control para lograr la eficacia del SGC.

De acuerdo con los resultados obtenidos, se focalizan las necesidades de la organización en miras de fortalecer el sistema de gestión de la calidad e incorporar el concepto integral bajo las normas ISO 14001 y OSHAS 18001 dentro de la organización. A partir de estas necesidades, se realiza el diseño de la guía del sistema de gestión integral para la organización Óptima de Urabá bajo la estructura de alto nivel.

Dentro de los requisitos propuestos en esta guía, se requirió de un trabajo minucioso que lograra acoplar las necesidades y oportunidades de la organización con las 
normas de gestión de una manera integral, para esto se tuvo en cuenta la teoría de gestión integral estipulada en el marco referencial, el cual ayudó a tener más claridad en la estructuración y en la metodología de la guía.

La guía fue ajustada a las características propias de la organización, en miras de dar respuesta específica a los hallazgos reportados en el diagnóstico y a la naturaleza específica de la organización asociada al sector de servicios públicos, las cuales tiene unas particularidades (en especial normativas) que fueron contempladas. Así mismo, se incorpora dentro de la guía los nuevos enfoques de los requisitos de las normas ISO 9001:2015 asociadas al análisis del contexto (interno y externo), determinación de las partes interesadas, gestión del cambio, gestión del conocimiento (específico de ISO 9001:2015) y enfoque de ciclo de vida (específico de ISO 14001:2015). Cabe resaltar que los aportes realizados por los expertos en el proceso de validación de la guía denotan un valor de peso más influyente, debido a que son los autores de referencia de la teoría en la cual se basó la construcción de la guía.

Los resultados de la investigación se encuentran alineados a la metodología establecida, donde los mecanismos implementados posibilitaron estandarizar la estructura de gestión integral articulando los requisitos de las tres normas de gestión ISO 9001, ISO 14001 y OHSAS 18001; así mismo, las teorías adoptadas aportaron mucho al diseño metodológico de la investigación, donde cada una de ellas permitieron medir la aplicabilidad de las herramientas implementadas.

Con respecto a la hipótesis planteada "eficacia de la guía para la implementación de un sistema de gestión integral para la empresa Óptima de Urabá", la guía trata de abordar de manera precisa los conceptos relacionados con el conocimiento de la organización, gestión del cambio, determinación de las condiciones ambientales externas de la organización, roles y responsabilidades y gestión del talento humano, de manera que se ajustará más a las necesidades de la organización y a la consecución de la eficacia del SGI.

Finalmente, la investigación da cumplimiento al objetivo general con la entrega de la Guía del SGI para la empresa Óptima de Urabá, la cual hace un aporte valioso a la organización e imparte beneficios para el fortalecimiento de su sistema de gestión actual. Paralelamente, se considera que suministra grandes herramientas de gestión al sector de servicios públicos y a la comunidad académica, debido al esquema de integración propuesto en este tipo de organizaciones.

\section{REFERENCIAS}

Álvarez, R. F. (2014). Estructura de alto nivel de la ISO y su impacto en las normas de sistemas de gestión. Cegesti Lexito Empresarial, 1(227), 1-3.

Atehortúa, F., Bustamante, R., y Valencia, J. (2008). Sistema de Gestión Integral. Una sola gestión, un solo equipo. Bogotá D. C.: Editorial Gestión y Conocimiento S.A.S.

Benavides, S. G. (2013). Ética empresarial y sistemas integrados de gestión: organizaciones pioneras certificadas en Bogotá, D. C. Signos, 5(2), 71-86.

BSI, B. S. (2015). Documento técnico Introducción al Anexo SL. La mueva estructura de alto nivel para todas las futuras normas de sistemas de gestión. Group BSI, Ref. UK, 569, SC/0215, BLD. Recuperado de https:// www.bsigroup.com/LocalFiles/es-ES/Documentos\%20tecnicos/Revisiones\%20ISO/ISO\%209001/ BSI-Anex0\%20SL-ISO-9001-2015.pdf

Gerard, B. (2014). Manual práctico para la implantación del estándar OHSAS 18001. Madrid, España: 
Majadahonda. Recuperado de http://prevencion.fremap.es/Buenas\%20prcticas/LIB.019\%20-\%20Manual\%20implantacion\%200HSAS\%2018001.pdf

Losada, F. T., y Guarin, G. P. (2009). Reflexiones sobre las caracteristicas constitutivas de la gestión integral. Signos, 5(2), 79-93.

Morín, E. (1994). Ciencia con conciencia. Pensamiento criticolpensamiento utópico. Investigación en sistemas de gestión. Signos, 5(1), 31-47. Barcelona: Antrophos Editorial.

Ortiz, P. A. (2014). La gestión integrada de los procesos en universidades. Procedimiento para su evaluación. Ingenieria Industrial, 36(1), 91-103.

Parasuraman A, Z. V. (1985). A conceptual model of service quality and its implications for future research. Journal of Marketing, 49(4), 41-50.

Salomone, R. (2011). Integrated management systems: Experiences in italian organizations. Journal of Cleaner Prodution, 16(1), 1786-1806.
Sampieri, R. H., Fernández, C. C., y Baptista, L. P. (2014). Metodologia de la Investigación. México: McGraw-Hill Interamericana.

Savino, M. M. (2015). Investigating the resources for Integrated Management Systems within resourcebased and contingency perspective in manufacturing firms. Journal of cleaner production, 104, 392402. ISSN: 0959-6526.

Urquijo, J. G. (2012). El sistema de gestión de la calidad como modelo de cambio organizacional en el sector público colombiano. Signos, 4(1), 15-33.

Valencia, P., Madrid, H. L., y Tabares, S. J. (2014). Inversiones en infraestructura versus comportamiento de pago de los clientes en Aguas de Urabá S. A. E.S.P. (Tesis de especialización). Medellín: Universidad de San Buenaventura. 